\section{Derivation of the \\ Fast Pole-Zero (ARMA) Recursive Least Squares \\ Algorithm \\ Using Geometric Projections}

by

Sasan H. Ardalan

Center for Communications and Signal Processing Department of Electrical and Computer Engineering North Carolina State University

Box 7914

Raleigh, NC 27695-7914

February 1985

CCSP-TR-85/3 


\section{ABSTRACT}

The Fast Pole-Zero (ARMA) Recursive Least Squares Algo$r$ ithm is derived using geometric projections. The new algorithm has a reduced number of computations compared to the algorithm obtained using matrix methods. In particular two inner products are eliminated. This is particularly important in multiprocessor implementations of the algorithm. The geometric approach provides insight and useful interpretations of the various filters and residuals that form the algorithm. Such insights are absent in the matrix derivation. Simulations verifying the performance of the algorithm are presented where the poles and zeroes of an IIR filter are estimated. The algorithm finds application in echo cancellation and system identification. 
1. Introduction

The Recursive Least Squares algorithm has been applied to a wide range of adaptive filtering problems. In many of these applications the system impulse response is estimated. In these cases the RLS algorithm has the structure of transversal filters and fast estimation schemes have been derived for efficient solution of these problems. These include the work by [1] and [2]. In the latter work the RLS problem is formulated geometrically and using geometric projections efficient solutions are derived. The resulting algorithms have fewer computations than the fast Kalman algorithm derived in [3] and used in [1]. Furthermore, the geometric approach provides insight into the interpretation and significance of the various filters and quantities originally encountered in [3].

Recently the RLS algorithm was applied to echo cancellation using pole-zero modeling $[4,5]$. In this case the fast Kalman algorithm derived in 3 is formulated to include the echo in addition to the echo generation signal to estimate the parameter vector containing the numerator and denominator coefficients of the echo path transfer function. In other words the echo path is modeled as an ARMA (pole-zero) process. The excellent performance of this algorithm in achieving very rapid and extremely high echo cancellation $[6,4]$ has provided motivation for deriving a more efficient and robust algorithm. 
In this paper, the geometric approach used in $[7,8]$ and [2] is extended to the derivation of a fast pole-zero (ARMA) Recursive Least Squares algorithm。 The work is also an extension of the formulation presented in [3] where a fast estimation scheme is developed using permutation matrices. The pole-zero algorithm differs from the multichannel Fast Transversal Filter of [2] in that the order of poles and zeroes can be independently and arbitrarily specified. However, the same efficiency in terms of the elimination of some vector inner products and other operations in comparison with the fast Kalman algorithm are achieved as in the case of the Fast Transversal Filter.

Also in this paper, an alternative derivation of the projection update formula is presented using matrix methods.

\section{Pole Zero Modeling}

In the pole zero modeling of the linear system $S$, the transfer function $H(z)$ is written as,

$$
H(z)=\frac{Y(z)}{X(z)}=\frac{\sum_{i=0}^{i=M} b_{i} z^{-i}}{1+\sum_{i=1}^{i=L} a_{i} z^{-i}}
$$

Hence we can write in the discrete time domain,

$$
y(n)=\sum_{i=0}^{i=M} b_{i} x(n-i)-\sum_{i=1}^{i=L} a_{i} y(n-i)
$$


Define the vector $(N=M+L)$, note that " " denotes the transpose of a vector,

$\underline{z}(n)=[x(n) x(n-1) \ldots x(n-M) y(n-1) \ldots y(n-L)]^{\prime} \quad N x I$

and

$\mathrm{w}_{\mathrm{N}}^{*}=\left[\mathrm{a}_{0} \mathrm{a}_{1} \ldots \mathrm{a}_{\mathrm{M}}-\mathrm{b}_{1} \ldots-\mathrm{b}_{\mathrm{L}}\right]^{\prime}$

Then we can write $y(n)$ in vector notation as an inner product,

$y(n)=\underline{z}^{\prime}(n) w_{N}^{*}$

3. Geometric Projection Formulation

The solution to the RLS adaptive-filtering problem is stated as any vector $\mathrm{W}_{\mathrm{N}}, \mathrm{T}$ for which

$\xi_{N}(T)=\sum_{t=0}^{T}\left[d(t)+W_{N}, T \underline{z}(t)\right]^{2}$

is minimized. In the case of pole/zero modeling the desired signal $d(t)=y(t)$ the current system output sample.

Define the following vectors,

$x_{T}=[x(T) x(T-1) \ldots x(0) 0 \ldots 0]^{\prime}, \quad J x 1$

$y_{T}=[y(T-1) y(T-2) \ldots y(0) 0 \ldots 0]^{\prime} \quad J x I$

The above vectors group all measurements of $x(n)$ and $y(n-1)$ up to time $T$ into vectors. Define,

$$
\begin{aligned}
& x_{M, T}=\left[x_{T} x_{T-1} \cdots x_{T-M+1}\right] J x M \\
& Y_{L, T}=\left[y_{T} Y_{T-1} \cdots y_{T-L+1}\right] J x L
\end{aligned}
$$


$z_{N, T}=\left[x_{T} x_{T-1} \cdots x_{T-M+1} y_{T-1} \cdots y_{T-L}\right] \quad J x(M+L) ; J x N$

$Z_{N, T}=\left[X_{M, T} Y_{L, T}\right] \quad J X N$

Note that $\mathrm{N}=\mathrm{M}+\mathrm{L}$. Furthermore define the vector,

$z_{T}=\left[x_{T} y_{T}\right] J \times 2$

which collects all measurements into a single vector. Also define the vector of desired response,

$d_{T}=[d(T) d(T-1) \ldots d(0) 0 \ldots 0]^{T}$

Hence the prediction error becomes,

${ }_{N, T}=d_{T}+Z_{N, T} T_{N, T}^{\prime}$

From which,

$\xi_{N}(T)=\varepsilon_{N}^{\prime}, T^{\varepsilon} N, T$

In a vector space interpretation the minimizing solution is the $W_{N, T}$ that forms a linear combination of the input vectors

$$
z_{\mathrm{T}}, \mathrm{z}_{\mathrm{T}-1}, \ldots, \mathrm{z}_{\mathrm{T}-\mathrm{N}-1}
$$

which has a minimum distance from the desired response vector, $d_{T}$. In the vector space $\varepsilon_{N}, T$ is orthogonal to the subspace that spans all the linear combinations of the $\mathrm{N}$ most recent input vectors. That is, ${ }{ }_{N}, T$ is orthogonal to the column space of $Z_{N, T}$ and thus satisfies,

$z_{T-i^{\varepsilon} N, T}^{\prime}=\left[\begin{array}{ll}0 & 0\end{array}\right]$ for $i=0, \ldots, N-1$.

In matrix notation this becomes, 
$Z_{N, T^{\varepsilon} N, T}^{\prime}=Z_{N, T}^{\prime}\left(d_{T}+Z_{N, T} W_{N, T}^{\prime}\right)=0_{N}$.

The solution is,

$W_{N, T}=-d_{T}^{\prime} Z_{N, T}\left(Z_{N, T}^{\prime} Z_{N, T}\right)^{-1}$

As pointed out in [2] if the sample autocorrelation matrix is singular, a situation that always occurs when the discrete time index $\mathrm{T}$ is less that $\mathrm{N}-1$, then any generalized inverse yields a solution to the RLS problem. We can write,

${ }_{N, T}=\left[I-Z_{N, T}\left(Z_{N, T}^{\prime} Z_{N, T}\right)^{-1} Z_{N, T}^{\prime}\right] d_{T}$

Define the projection operators,

$P_{z}=z\left(z^{\prime} z\right)^{-1} z^{\prime}$

and,

$\mathrm{P}^{\prime}=\mathrm{I}-\mathrm{P}_{\mathrm{Z}}$

Hence,

$\varepsilon_{N, T}=P_{N, T}^{\mid} d_{T}$

The matrix ${ }_{N}, T$ projects vectors onto the subspace spanned by the columns of $z$, i.e. the past observations. The projection operator $\mathrm{P}_{\mathrm{N}, \mathrm{T}}$ is its orthogonal complement and projects vectors onto a subspace that is orthogonal to the subspace associated with $\mathrm{P}_{\mathrm{N}, \mathrm{T}}$. In other words [2], the prediction error ${ }_{\varepsilon_{T}}=P_{N, T}^{l} d_{T}=d_{T}-\hat{d}_{T}$ is orthogonal to the predicted estimate $d_{T}=P_{N}, T d_{T}$.

An important property of the projection operator is the idempotent property: 
$\mathrm{P}_{\mathrm{N}, \mathrm{T}}^{2}=\mathrm{P}_{\mathrm{N}, \mathrm{T}}$

We also define the quantity,

$K_{z}=z\left(z^{\prime} z\right)^{-1}$

From which using $(3.14)$ we can write the optimum weights as,

$W_{N, T}=-d_{T}^{\prime} K_{N}, T$

Therefore, $U^{\prime} K_{Z}$ can be interpreted [2] as the linear transformation which acts on the subspace $z$ to yield the closest approximation to $U$ in the least-squares or minimum distance sense. In other words $U^{\prime} K_{z}$ is a linear filter that acts on the input vectors to estimate $U$. In our case $U=d_{T}$. Also, $K_{Z}$ can be interpreted as a generalized $r i g h t$ inverse for $\mathrm{Z}$ :

$$
z^{\prime} K_{Z}=I
$$

Furthermore,

$$
P_{Z} K_{Z}=K_{Z}
$$

As in $[7,9]$ and [2] we define the pinning vector,

$\left.\sigma=\left[\begin{array}{llllll}1 & 0 & 0 & \ldots & 0\end{array}\right]^{\prime} \mathrm{Jx}\right]$.

The pinning vector has the important property that its inner product with the time vectors $d_{T}{ }^{\varepsilon}{ }_{N}, T$, and $z_{T}$ yields the most recent sample:

$$
\begin{aligned}
& \sigma^{\prime} \mathrm{d}_{\mathrm{T}}=\mathrm{d}(\mathrm{T}) \\
& \sigma^{\prime} \mathrm{z}_{\mathrm{T}}=[x(n) y(n-1)]^{\prime}
\end{aligned}
$$


$\sigma^{\prime} \varepsilon_{\mathrm{N}, \mathrm{T}}=\varepsilon(\mathrm{T})$

4. Filters for RLS Algorithm

We introduce the following filters for the pole/zero RLS algorithm:

$$
\begin{array}{lll}
A_{N}, T=\left[\begin{array}{lll}
I_{2} & -z_{T}^{\prime} K_{N}, T-1
\end{array}\right] & 2 x(N+2) \\
B_{N, T}=\left[\begin{array}{lll}
-z_{T-N} K_{N}, T & I_{2}
\end{array}\right] & 2 \times(N+2) \\
C_{N, T}=-\sigma K_{N}, T & 1 \times N & \\
W_{N, T}=-d_{T} K_{N}, T & 1 \times N &
\end{array}
$$

Corresponding to these filters are the residuals obtained by appropriately exciting these filters. First define

$$
\begin{aligned}
& \underline{\underline{z}}(T)=[x(T) x(T-1) \ldots x(T-M+1) y(T-1) \ldots y(T-L+1)]^{\prime} \\
& \varphi_{F} \bar{z}(T)=[x(T) \quad y(T-1) \quad \underline{z}(T-1)]^{\prime}
\end{aligned}
$$

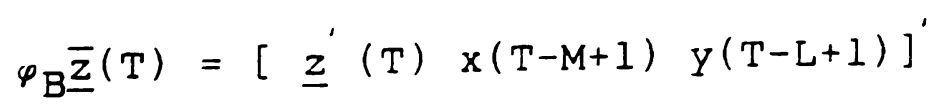

The permutation matrices $\varphi_{B}$ and $\varphi_{F}$ were introduced in [3] and have the important property that

$$
\varphi \varphi^{\prime}=I
$$

Now,

$$
\begin{array}{ll}
e_{N}(T)={ }_{N} T_{T^{p}} \underline{z}^{\prime}(T)=\underline{z}^{\prime}(T) P_{N, T-I^{\sigma}}^{l} & 2 \times 1 \\
r_{N}(T)=B_{N}, T-1{ }^{\varphi} B^{\prime} &
\end{array}
$$


${ }_{N}(T)=1+C_{N, T} \underline{z}(T)$

${ }_{N}(T)=\sigma^{\prime} P_{N_{\theta}}^{l} T^{\sigma}$

${ }^{\varepsilon}{ }_{N}(T)=d_{T}^{\prime} P_{N}^{l}, T^{\sigma}$

The above quantities can be interpreted as the filtered residuals. In particular (4.10) provides an interesting interpretation for $C_{N}, T$ as the filter which when excited forms the best estimate of the quantity one. The residual being $\gamma_{N}(T)$. In [7] the term $\gamma_{N}(T)$ is seen to be the cosine squared of the angle between $\underline{z}(T)$ and its projection on the space $z_{N, T-1}$. Hence as $\gamma_{N}(T)$ approaches one the gap between the spaces closes. In other words it is a measure of how much new information is provided.

We define the prediction residuals as,

$$
\begin{aligned}
& e_{N}^{p}(T)=A_{N}, T-I^{\varphi} B^{\bar{z}}(T) \quad 2 \times 1
\end{aligned}
$$

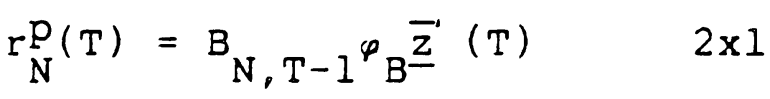

$$
\begin{aligned}
& \varepsilon_{\mathrm{N}}^{\mathrm{p}}=\mathrm{d}(\mathrm{T})+\mathrm{W}_{\mathrm{N}, \mathrm{T}-\mathrm{I}} \underline{\mathrm{z}}^{\prime}(\mathrm{T})
\end{aligned}
$$

Define the residual powers,

$$
\begin{array}{ll}
\alpha_{N}(T)={ }_{N}, T^{\varphi} F_{N} Z_{N} T^{Z_{N}^{\prime} T^{\varphi} F^{A}{ }_{N}^{\prime} T} & 2 \times 2 \\
\beta_{N}(T)=B_{N}, T^{\varphi} B_{N} Z_{N} T^{Z_{N}^{\prime} T^{\varphi}{ }_{B} B_{N}^{\prime} T} & 2 \times 2
\end{array}
$$

Or, using the idempotent property of the projection operators, 
$a_{N}(T)=z_{T}^{\prime} P_{N} \mid T-z_{T}$
${ }^{\beta}{ }_{N}(T)=z_{T-N}^{\prime} P_{N} \mid T_{T} z_{T-N}$

Furthermore,

$\gamma_{\mathrm{N}}(\mathrm{T})=\left\|\sigma+\mathrm{Z}_{\mathrm{N}, \mathrm{T}} \mathrm{C}_{\mathrm{N}, \mathrm{T}}^{\prime}\right\|^{2}=\sigma^{\prime} \mathrm{P}_{\mathrm{N}, \mathrm{T}^{\sigma}}^{\prime}$

$\xi_{N}(T)=|| d_{T}+Z_{N},\left.T_{N} W_{N}^{\prime} T\right|^{2}=d_{T}^{\prime} P_{N}^{\prime}, T^{d_{T}}$

5. Projection Operator and Filter Updating

There are two types of projection operator updating which for the case of the pole/zero RLS have to be distinguished. One involves order updating and the other involves time updating. In order updating, the order of the projection operator is increased by augmenting a new column space as follows:

$P_{Z, W}=\left[\begin{array}{ll}Z & W\end{array}\right] \varphi_{B}^{\prime}\left\{\varphi_{B}\left[\begin{array}{l}Z \\ Z\end{array}\right]\left[\begin{array}{ll}Z & W\end{array}\right]{ }^{\prime} \varphi_{B}^{\prime}\right\}^{\#} \varphi_{B}\left[\begin{array}{l}Z^{\prime} \\ W^{\prime}\end{array}\right] \varphi_{B}^{\prime}$

Or,

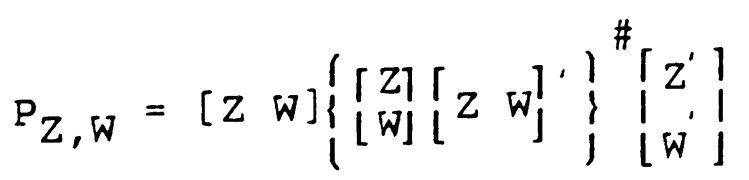

In case of order updating $\mathrm{Z}=\mathrm{Z}_{\mathrm{N}, \mathrm{T}}$ and $\mathrm{W}=\mathrm{z}_{\mathrm{T}-\mathrm{N}}$. For time updating $W=\sigma$ as will be shown. The projection update can be shown to be [8] (also see appendix for a matrix derivation),

$\mathrm{P}_{\mathrm{Z}, \mathrm{W}}=\mathrm{P}_{\mathrm{Z}}+\mathrm{P}_{\mathrm{Z}} \mathrm{W}^{\mathrm{W}}\left(\mathrm{W}^{\prime} \mathrm{P}_{\mathrm{Z}} \mathrm{W}^{\# \mathrm{~W}^{\prime} \mathrm{P}} \mathrm{Z}\right.$

Define, 


$$
\mathrm{V}=\mathrm{P}_{\mathrm{Z}}^{\mathrm{l}} \mathrm{W}
$$

Then,

$\mathrm{P}_{\mathrm{Z}, \mathrm{W}}=\mathrm{P}_{\mathrm{Z}}+\mathrm{P}_{\mathrm{V}}$

The update for the orthogonal projection becomes,

$\mathrm{P}_{\mathrm{Z}, \mathrm{W}}^{\mid}=\left(\mathrm{P}_{\mathrm{Z}}^{\mid}-\mathrm{P}_{\mathrm{Z}}^{\mid} \mathrm{W}\left(\mathrm{W}^{\prime} \mathrm{P}_{\mathrm{Z}}^{\mid} \mathrm{W}^{\#}{ }^{\#} \mathrm{~W}^{\prime} \mathrm{P}_{\mathrm{Z}}\right)\right.$

For filter updating we note that,

$\varphi_{B}\left\{\begin{array}{l}\left.Z^{\prime} W^{\prime}\right\} K_{Z, W} \\ I_{Z, W}\end{array}\right.$

Also,

$\mathrm{P}_{\mathrm{Z}, \mathrm{W}} \mathrm{K}_{\mathrm{Z}, \mathrm{W}}=\mathrm{K}_{\mathrm{Z}, \mathrm{W}}$

Define,

$\hat{\mathrm{K}}_{\mathrm{Z}, \mathrm{W}}=\mathrm{K}_{\mathrm{Z}, \mathrm{w}^{\varphi} \mathrm{B}}$

and,

$\tilde{\mathrm{K}}_{\mathrm{W}, \mathrm{Z}}=\mathrm{K}_{\mathrm{W}, \mathrm{Z}^{\varphi} \mathrm{F}}$

Then substituting for $P_{Z, W}$ we obtain

$\hat{\mathrm{K}}_{Z, W}=\mathrm{P}_{Z^{\mathrm{K}} \mathrm{Z}, \mathrm{W}}+\mathrm{P}_{\mathrm{Z}}^{\mid} \mathrm{W}\left(\mathrm{W}^{\prime} \mathrm{P}_{\mathrm{Z}}^{\mid} \mathrm{W}^{\#}{ }_{\mathrm{W}^{\prime}}\left(\mathrm{I}-\mathrm{P}_{\mathrm{Z}}\right) \hat{\mathrm{K}}_{\mathrm{Z}, \mathrm{W}}\right.$

Or using (5.7),

$\hat{K}_{Z, W}=\left(\begin{array}{lll}K_{Z} & 0 & 0\end{array}\right)+P_{Z}^{\prime} W\left(W^{\prime} P_{Z}^{\prime}\right)^{\#}\left[\begin{array}{lll}-W^{\prime} & K_{Z} & I\end{array}\right]$

Similarly,

$\tilde{\mathrm{K}}_{\mathrm{W}, \mathrm{Z}}=\left[\begin{array}{lll}0 & 0 & \mathrm{~K}_{\mathrm{Z}}\end{array}\right]+\mathrm{P}_{\mathrm{Z}}^{\mid} \mathrm{W}\left(\left.\mathrm{W}^{\prime} \mathrm{P}_{\mathrm{Z}}\right|_{\mathrm{W}} \#\left[\begin{array}{ll}I & -\mathrm{W}^{\prime} \mathrm{K}_{\mathrm{Z}}\end{array}\right]\right.$. 


$$
\begin{aligned}
& P_{Z, W}=\left[P_{Z}+P_{Z}^{l} W\left(W^{\prime} P_{Z}^{l}{ }^{\prime}{ }^{\#} W^{\prime} P_{Z}^{l}\right]\right. \\
& U^{\prime} P_{Z, W}^{\prime} V=U^{\prime} P_{Z}^{\prime} V-\left(U^{\prime} P_{Z}^{\prime} W\right)\left(W^{\prime} P_{Z}^{\prime}\right)^{\prime}\left(W^{\prime} P_{Z}^{\prime} V\right)
\end{aligned}
$$

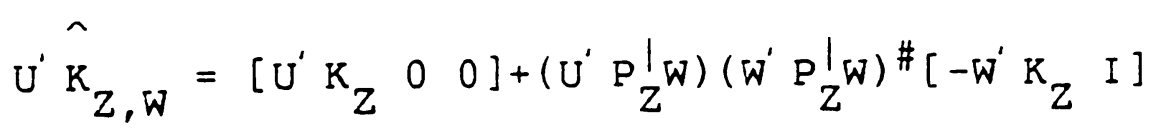

$$
\begin{aligned}
& U^{\prime} \tilde{K}_{W, Z}=\left[\begin{array}{lll}
0 & 0 & U^{\prime} K_{Z}
\end{array}\right]+\left(U^{\prime} P_{Z^{W}}^{\prime}\right)\left(W^{\prime} P_{Z^{W}}^{\prime}\right)^{\#}\left[\begin{array}{ll}
I & -W^{\prime} K_{Z}
\end{array}\right] \\
& \hat{\mathrm{K}}_{\mathrm{Z}, \mathrm{W}}=\mathrm{K}_{\mathrm{Z}, \mathrm{W}^{\varphi} \mathrm{B}} \\
& \tilde{\mathrm{K}}_{\mathrm{W}, \mathrm{Z}}=\mathrm{K}_{\mathrm{W}, \mathrm{Z}^{\varphi} \mathrm{F}} \\
& \hat{\mathrm{k}}_{W, \mathrm{z}}=\tilde{\mathrm{K}}_{\mathrm{W}, \mathrm{Z}^{\varphi} \mathrm{F}^{\prime}{ }_{\mathrm{B}}}
\end{aligned}
$$

\section{Derivation of Algorithm}

In the following derivation frequent use is made of the definitions of the filters and residuals of section 4 . The derivation follows [2] closely.

First we derive the time updates for the forward and backward prediction filters: ( use 5.3' with $\mathrm{W}=\sigma, \mathrm{U}=\mathrm{z}_{\mathrm{T}^{\prime}} \mathrm{Z}=\mathrm{Z}_{\mathrm{N}, \mathrm{T}-1}$ )

$A_{N, T-1}=A_{N, T}-\frac{e_{N}(T)}{\gamma_{N}(T-1)}\left[\begin{array}{lll}0 & 0 & C_{N}, T-1\end{array}\right]$

(use 5.4' with $U=z_{T-N}, W=\sigma, Z=Z_{N, T}$ ) 
$B_{N, T-1}=B_{N, T}-\frac{r_{N}(T)}{\gamma_{N}(T)}\left[C_{N}, T \quad 00\right]$

Order update $\mathrm{C}_{\mathrm{N}, \mathrm{T}-1}$ by using $\left(5.4^{\circ}\right)$ with $\mathrm{U}=\sigma, \mathrm{W}=\mathrm{z}_{\mathrm{T}}, \mathrm{Z}=\mathrm{Z}_{\mathrm{N}, \mathrm{T}-\mathrm{I}}$.

$C_{N+1, T}=\left[\begin{array}{llll}0 & 0 & C_{N, T-1}\end{array}\right]-e_{N}(T) a_{N}^{-1}(T) A_{N, T}$

Also using $\left(5.3^{\prime}\right)$ and $\mathrm{U}=\sigma, \mathrm{W}=\mathrm{z}_{\mathrm{T}-\mathrm{N}}, \mathrm{Z}=\mathrm{Z}_{\mathrm{N}, \mathrm{T}}$,

$\hat{C}_{N+1, T}=\left[\begin{array}{llll}C_{N, T} & 0 & 0 & ]+r_{N}^{\prime}(T) \beta_{N}^{-1}(T) B_{N, T}\end{array}\right.$

Close examination of (6.4) reveals that the last two elements of $\mathrm{C}_{\mathrm{N}+1, \mathrm{~T}}$ are equal to,

$\mu(T)=r_{N}^{\prime}(T) \beta_{N}^{-1}(T)$

So we can write

$\hat{\mathrm{C}}_{\mathrm{N}+1, \mathrm{~T}}=\left[\begin{array}{lll}\mathrm{C}_{\mathrm{N}, \mathrm{T}} & 0 & 0\end{array}\right]+\mu(\mathrm{T}) \mathrm{B}_{\mathrm{N}, \mathrm{T}}$

From $\left(5.2^{\prime}\right.$ ) using $U=V=z_{T}, W=\sigma, Z=Z_{N}, T-1$,

$\alpha_{N}(T)=\alpha_{N}(T-1)+e_{N}(T) \gamma_{N}^{-1}(T-1) e_{N}^{\prime}(T)$

Update $\beta_{N}(T)$ by using $\left(5.2^{\prime}\right)$ with $U=V=Z_{T-N}, W=\sigma, Z=Z_{N}, T$,

$\beta_{N}(T)=\beta_{N}(T-1)+r_{N}(T) \gamma_{N}^{-1}(T) r_{N}^{\prime}(T)$

To obtain the prediction errors post multiply (6.1) by $\varphi_{\mathrm{F}} \overline{\bar{z}}^{\prime}(\mathrm{T})$ and $(6.2)$ by $\varphi_{\mathrm{B}} \underline{\bar{z}}^{\prime}(\mathrm{T})$.

$e_{N}^{p}(T)=e_{N}(T)-e_{N}(T) \gamma_{N}^{-1}(T-1)\left(\gamma_{N}(T-1)-1\right)=\frac{e_{N}(T)}{\gamma_{N}(T-1)}$

$r_{N}^{p}(T)=\frac{r_{N}(T)}{r_{N}(T)}$ 
To obtain the order update for $\gamma_{N+1}(T)$ use (5.2') with $W=Z_{T-N^{\prime}} \quad Z=Z_{N}, T-I^{\prime} \quad U=V=\sigma$,
$\gamma_{N+1}(T)=\gamma_{N}(T)-r_{N}^{\prime}(T) \beta_{N}^{-1}(T) r_{N}^{(T)}$

Again in $\left(5.2^{\prime}\right)$ let $W=z_{T}, Z=Z_{N}, T-1, U=V=\sigma$,

$\gamma_{N+1}(T)=\gamma_{N}(T-1)-e_{N}^{\prime}(T) \alpha_{N}^{-1}(T) e_{N}(T)$

Define,

$\tilde{C}_{N, T}=\gamma_{N}^{-1}(T) C_{N}, T$

$\hat{C}_{N+1, T}=\gamma_{N+1}^{-1}(T) \varphi_{B} C_{N+1, T}$

$\tilde{\mathrm{C}}_{\mathrm{N}+1, \mathrm{~T}}=\gamma_{\mathrm{N}+1}^{-1}(\mathrm{~T}) \varphi_{\mathrm{F}} \mathrm{C}_{\mathrm{N}+1, \mathrm{~T}}$

Then from (6.3) substituting (6.1) and simplifying using (6.11) we obtain,

$C_{N+1, T}=\left[\begin{array}{llll}0 & 0 & C_{N}, T-1\end{array}\right]-e_{N}(T) a_{N}^{-1}(T) A_{N}, T-1-$

$e_{N}(T) \alpha_{N}^{-1}(T) e_{N}(T)\left[\begin{array}{lll}0 & 0 & C_{N}, T-1\end{array}\right]$

$C_{N+1, T}=\left[\begin{array}{lll}0 & 0 & C_{N}, T-1\end{array}\right]-$

$e_{N}(T) \alpha_{N}^{-1}(T) A_{N}, T-1\left[\gamma_{N+1}(T)-\gamma_{N}(T-1)\right] \gamma_{N}^{-1}(T-1)\left[\begin{array}{lll}0 & 0 & C_{N}, T-1\end{array}\right]$.

$C_{N+1, T}=\gamma_{N+1}(T) \gamma_{N}^{-1}(T-1)\left[\begin{array}{lll}0 & 0 & C_{N}, T-1\end{array}\right]-e_{N}(T) \alpha_{N}^{-1}(T) \gamma_{N+1}^{-1}(T) A_{N}, T-1$

Using the definition (6.11) we obtain,

$\hat{C}_{N+1, T}=\left[\begin{array}{lll}0 & 0 & \tilde{C}_{N}, T-1\end{array}\right]-e_{N}(T) a_{N}^{-1}(T) \gamma_{N+1}^{-1}(T) A_{N}, T-1$ 
Now from (6.11) through (6.13),

$\tilde{C}_{\mathrm{N}+1, \mathrm{~T}}=\hat{\mathrm{C}}_{\mathrm{N}+1, \mathrm{~T}^{\varphi} \mathrm{F}^{\varphi} \mathrm{B}}$

Substituting (6.2) into (6.4) we get,

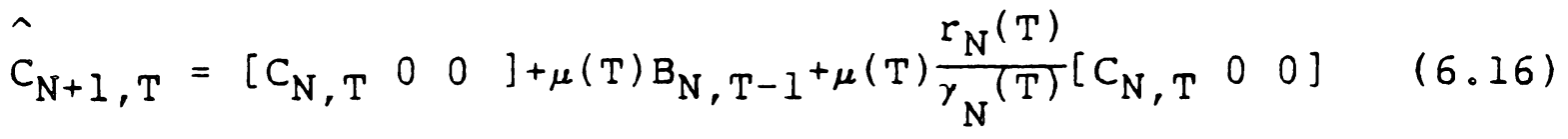

$$
\begin{aligned}
& \text { But } \mu(T)=\mathrm{r}_{N}^{\prime}(T) \beta_{N}^{-1}(T) \text {. Hence, using (6.9) we get, } \\
& C_{N+1, T}=\left[\begin{array}{lll}
C_{N, T} & 0 & 0
\end{array}\right]+\mu(T) B_{N, T-1}-\left[\gamma_{N+1}(T)-\gamma_{N}(T)\right] \frac{1}{\gamma_{N}(T)}\left[C_{N, T} 00\right] \\
& \left.\tilde{\mathrm{C}}_{\mathrm{N}+1, \mathrm{~T}}=\left[\begin{array}{lll}
\tilde{\mathrm{C}}_{\mathrm{N}, \mathrm{T}} & 0 & 0
\end{array}\right]+\tilde{\mu}_{\mathrm{N}} \mathrm{T}\right) \mathrm{B}_{\mathrm{N}, \mathrm{T}-1}
\end{aligned}
$$

Simultaneous solution of $(6.4)$ and (6.2) yields

$$
\left[C_{N}, T \quad 00\right]=\left[1+\mu(T) r_{N}^{p}(T)\right]^{-1}\left[C_{N+1, T^{-\mu}}(T) B_{N, T-1}\right]
$$

$$
\text { Excite by } \varphi_{\mathrm{B}} \overline{\mathrm{z}}_{\mathrm{T}} \text {. }
$$

$\gamma_{N, T}^{-1}(T)=\left[1+\mu(T) r_{N}^{p}(T)\right]^{-1}\left[\gamma_{N+1, T}^{-1}(T)-r_{N}^{\prime}(T) \beta_{N}^{-1}(T) r_{N}^{P}(T)\right]$

Substituting from (6.9) and simplifying,

$$
\gamma_{N}(T)=\left[1+\mu(T) r_{N}^{p}(T)\right]^{-1} \gamma_{N+1}(T)
$$

once $\tilde{C}_{N, T}$ has been updated then the joint process updates can be obtained:

$$
\begin{aligned}
& { }_{{ }_{N}}^{P}(T)=d(T)+W_{N}, T-1 \underline{z}(T) \\
& { }_{N}(T)=\varepsilon_{N}^{P}(T) \gamma_{N}(T) \\
& W_{N, T}=W_{N}, T-1+{ }^{\varepsilon} N(T) \tilde{C}_{N}, T
\end{aligned}
$$




$$
\begin{aligned}
& e_{N}^{p}(T)=A_{N}, T-1 \varphi_{F} \bar{z}(T) \\
& e_{N}(T)=e_{N}^{p}(T) \gamma_{N}(T-1) \\
& a_{N}(T)=a_{N}(T-1)+e_{N}^{p}(T) e_{N}^{\prime}(T) \\
& \gamma_{N+1}(T)=\gamma_{N}(T-1)-e_{N}^{\prime}(T) a_{N}^{-1}(T) e_{N}(T) \\
& \hat{\mathrm{C}}_{\mathrm{N}+1, T}=\left[\begin{array}{lll}
0 & 0 & \tilde{C}_{N, T-1}
\end{array}\right]-e_{N}^{\prime}(T) a_{N}^{-1}(T) \gamma_{N+1}^{-1}(T) A_{N, T-1} \\
& \tilde{\mathrm{C}}_{\mathrm{N}+1, \mathrm{~T}}=\hat{\mathrm{C}}_{\mathrm{N}+1, \mathrm{~T}} \hat{\varphi}_{\mathrm{F}^{\varphi} \mathrm{B}} \\
& A_{N, T}=A_{N, T-1}+e_{N}(T)\left[\begin{array}{lll}
0 & 0 & \tilde{C}_{N, T-1}
\end{array}\right] \\
& \mathrm{r}_{\mathrm{N}}^{\mathrm{P}^{\prime}}(\mathrm{T})=\tilde{-\mu}(\mathrm{T}) \beta_{\mathrm{N}}(\mathrm{T}-1) \\
& \text { Note that } \tilde{\mu}(T)=\tilde{C}_{N+1}^{N} \text { is the last two elements of } \tilde{\mathrm{C}}_{N+1, T} \text {. } \\
& \gamma_{N}(T)=\left[1+\mu(T) r_{N}^{p}(T) \gamma_{N+1}(T)\right]^{-1} \gamma_{N+1}(T) \\
& r_{N}(T)=r_{N}^{p}(T) \gamma_{N}(T) \\
& \beta_{N}=\beta_{N}(T-1)+r_{N}^{p}(T) r_{N}^{\prime}(T) \\
& {\left[\tilde{\mathrm{C}}_{\mathrm{N}, \mathrm{T}}\left[\begin{array}{lll}
0 & 0
\end{array}\right]=\tilde{\mathrm{C}}_{\mathrm{N}, \mathrm{T}}-\tilde{\mu}^{(\mathrm{T}) \mathrm{B}_{\mathrm{N}}, \mathrm{T}-1}\right.} \\
& B_{N, T}=B_{N, T-1}+r_{N}(T)\left[\tilde{C}_{N, T} 000\right]
\end{aligned}
$$


16

Joint-Process Extension.

$$
\begin{aligned}
& \varepsilon_{N}^{p}(T)=d(T)+W_{N, T-I}(T) \\
& { }^{\varepsilon}{ }_{N}(T)={ }_{\varepsilon}{ }_{N}^{p}(T) \gamma_{N}(T) \\
& W_{N, T}=W_{N, T-1}+\varepsilon_{N}(T) \tilde{C}_{N, T}
\end{aligned}
$$




\section{Simulation Results}

In this section the results of simulating the Fast Pole-zero Recursive Least Squares algorithm, derived and summarized in the previous section, are presented. The algorithm was programmed in $C$. To test the algorithm the poles and zeroes of a loth order elliptic bandpass digital filter were estimated. The pole-zero distribution of the filter is shown in figure 1 . In figure 2 the estimated poles and zeroes using the algorithm when a white Gaussian noise sequence is used to excite the filter is shown. Note the close estimation of the pole and zero locations. Figure 3 shows the prediction error plotted in $\mathrm{dB}^{\prime} \mathrm{s}$ against the number of iterations. Note the rapid convergence of the algorithm. Also the prediction error is more than $100 \mathrm{~dB}$ 's below the desired signal, an indication of the high degree of accuracy achievable by pole-zero modeling in comparison to impulse response modeling. In an echo cancellation application this corresponds to a $100 \mathrm{~dB}$ echo return loss enhancement. Where appropriate and as a measure of the algorithm's performance the ratio of the power of the desired signal over the power of the prediction error is given in dB's. Ideally, in the absence of noise, this ratio is very large. In all cases the first $\mathrm{N}$ iterations are discarded.

The effect of zero overspecification is presented in figures 4 and 5 . In this case the number of zeroes was 
overspecified by $10 \quad(M=20, L=10)$. The zeroes are distributed around the origin and two of them cancel out two of the poles. The rapid convergence and high degree of prediction accuracy is again observed in figure 4 with a slight degradation in comparison with no zero overspecification.

The interesting case of both pole and zero overspecification is presented in figure 6 $(M=20, L=20)$. Notice that the extra poles and zeroes cancel each other out.

An important property of the algorithm is its ability to predict the desired signal in the presence of noise. In figure 7 , the prediction error is plotted against the number of iterations for the case where white Gaussian noise with an rms value of 0.1 was added to the desired signal. Note that the prediction error is uncorrelated in contrast to the filtered and correlated desired signal. In other words the desired signal has been eliminated from the prediction error. In an echo cancellation application this corresponds to the elimination of the echo even in the presence of noise. The pole-zero distribution for this case is shown in figure 8. Note that noise does effect the location of the poles and zeroes. In fact, since the noise contaminates $y(n)$ which is used in the prediction of the desired signal a bias is introduced in the estimation of the weight vector $\mathrm{W}_{\mathrm{N}, \mathrm{T}}$. 


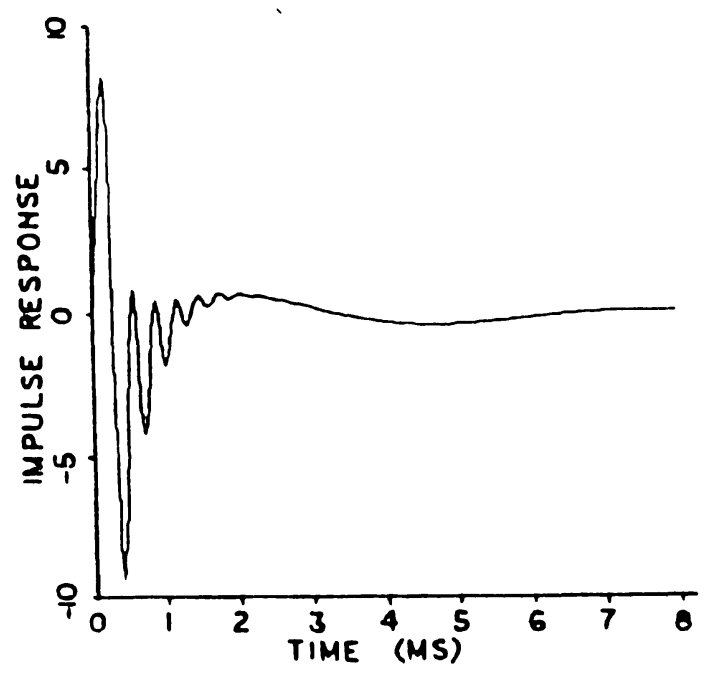

Digltal IIR Filter Impulse Response

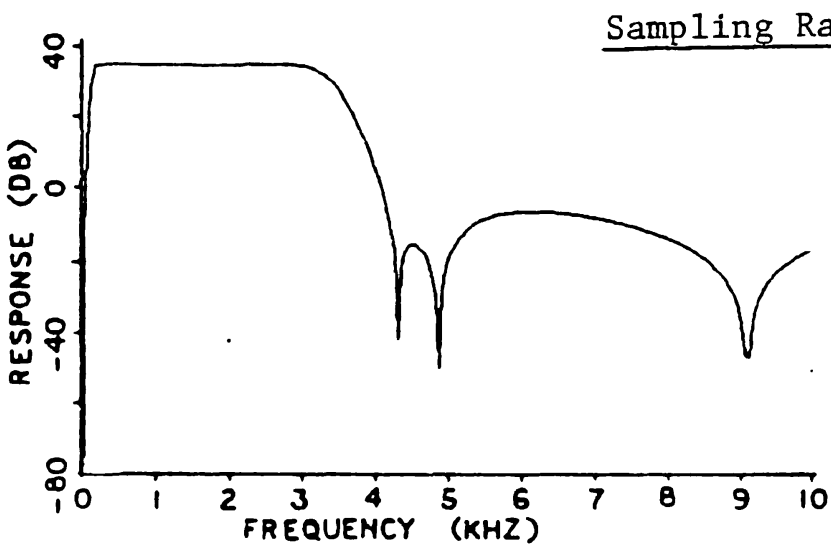

Digital IIR F1lter Frequency Response

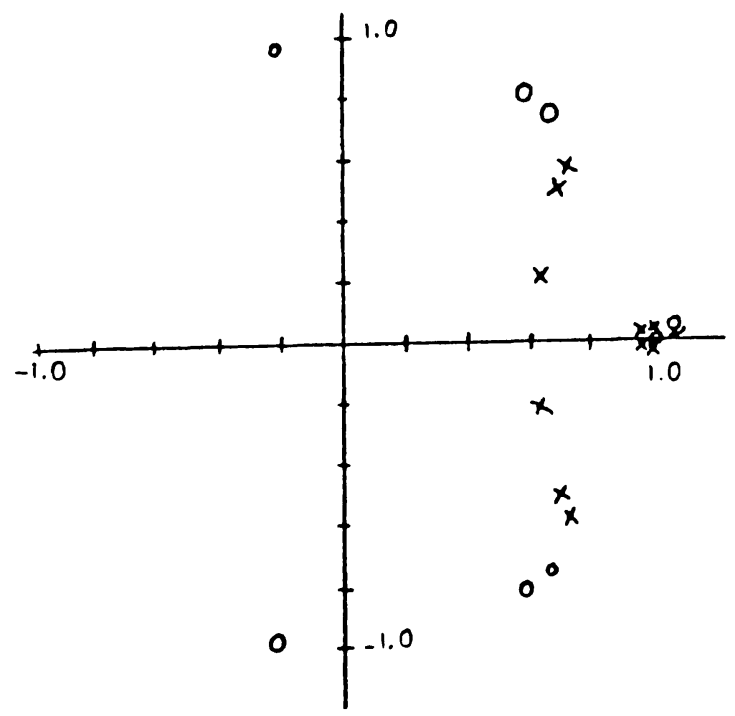

Fig. 1 Pole-zero Distribution of IIR Digital Filter 


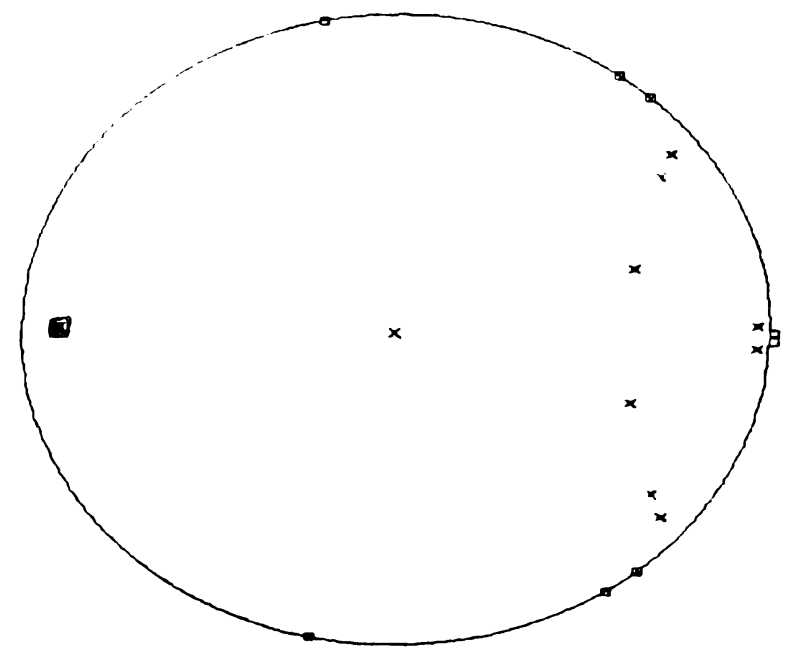

Fig. 2 Estimated Pole-Zero Distribution $(M=10, L=10)$

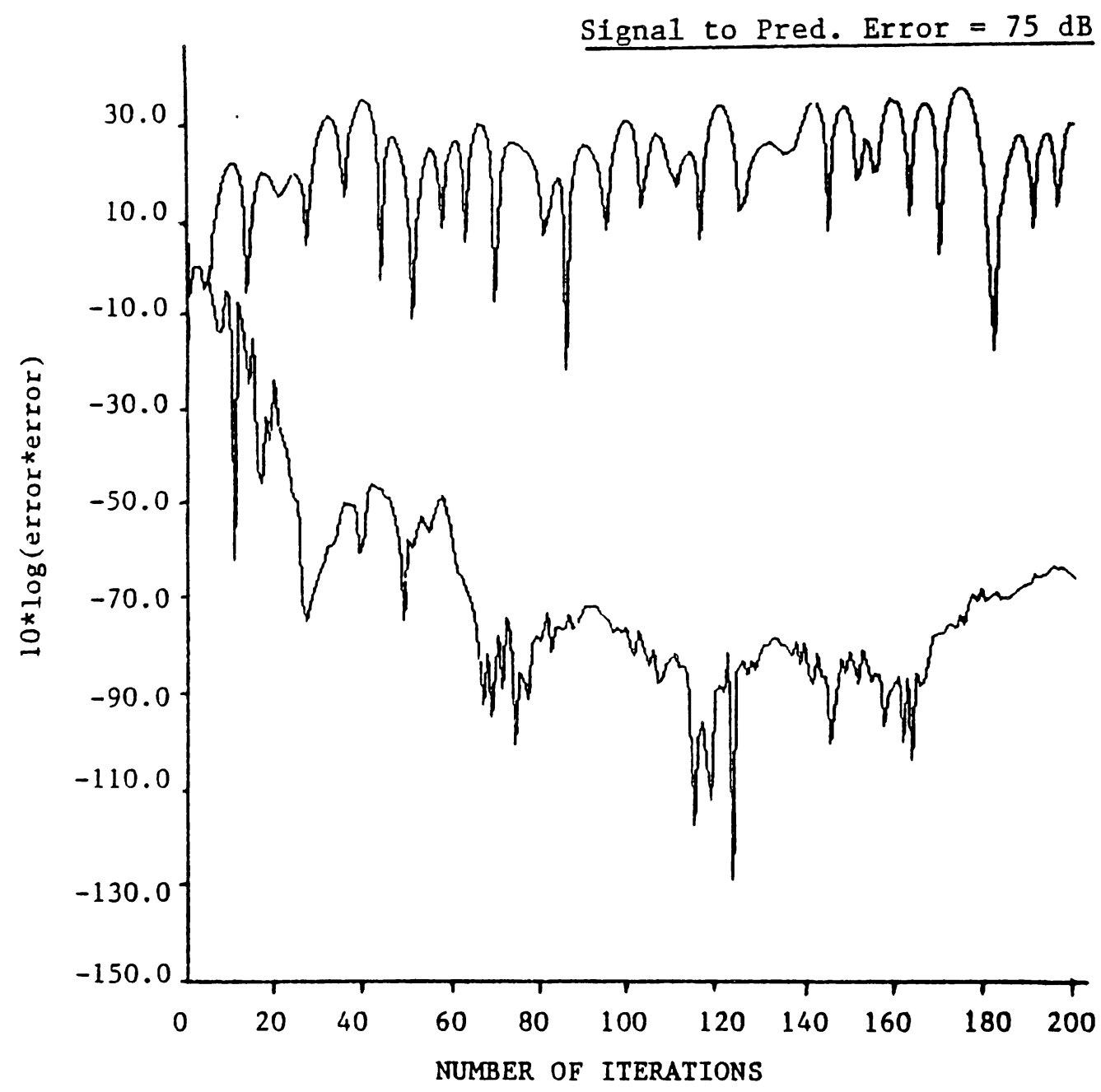

Fig. 3 Convergence of Fast Pole-Zero Algorithm $(M=10, L=10)$ 


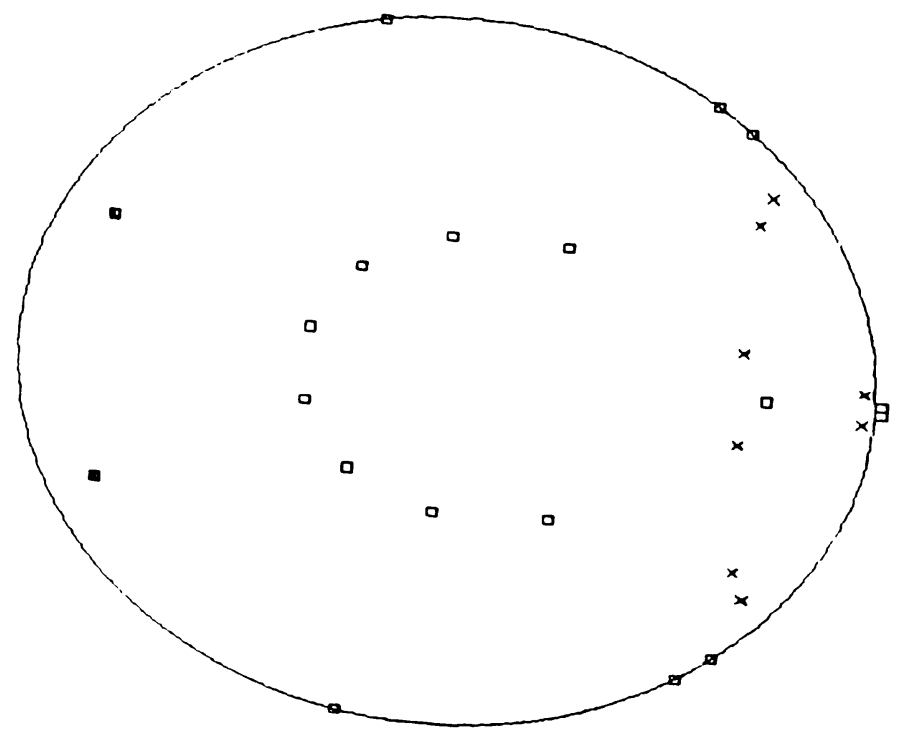

Fig. 4 Pole-Zero Distribution Zero Overspecification $(M=20, L=10)$

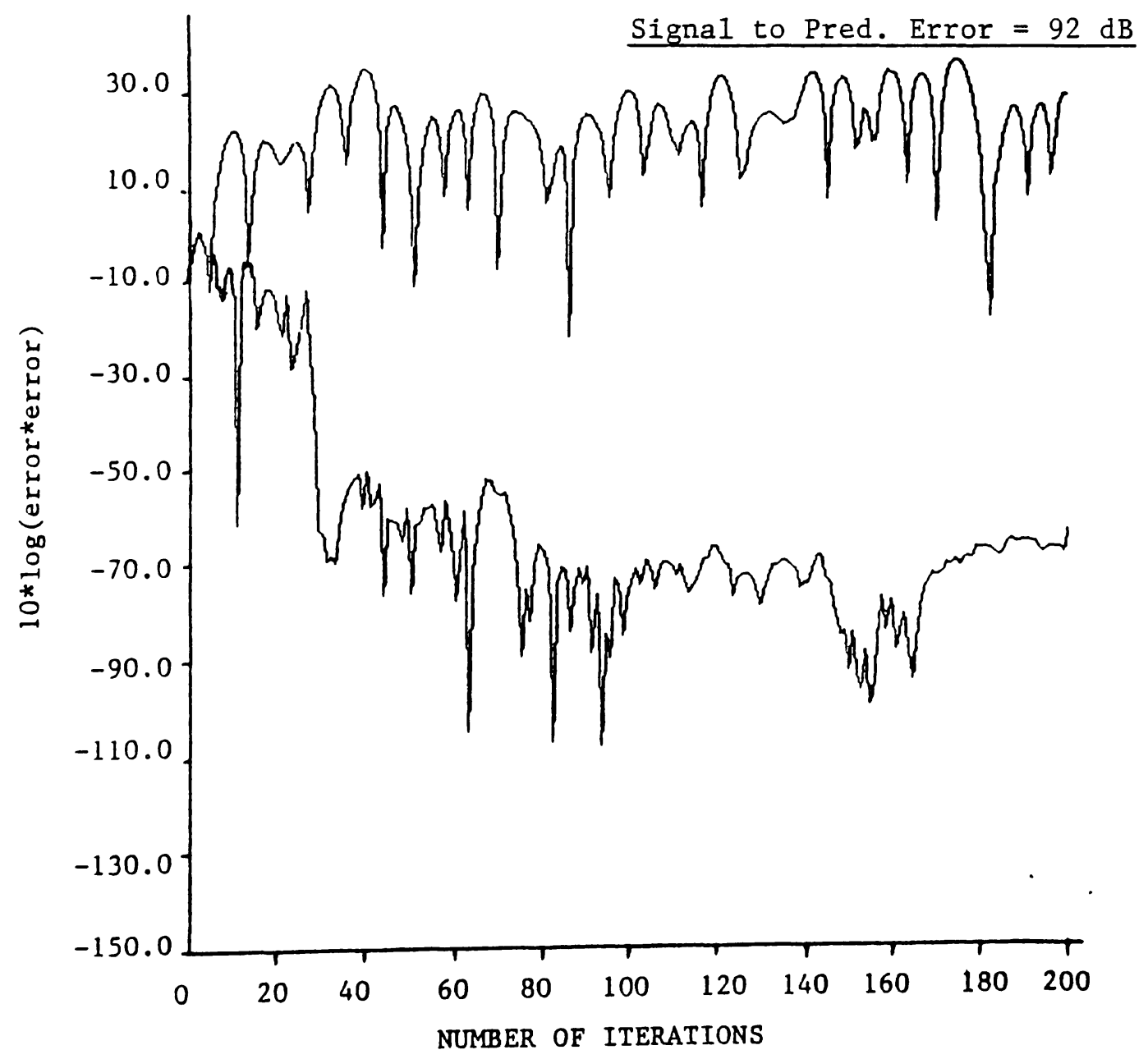

Fig. 5 Convergence with zero over Specification $(M=20, L=10)$ 


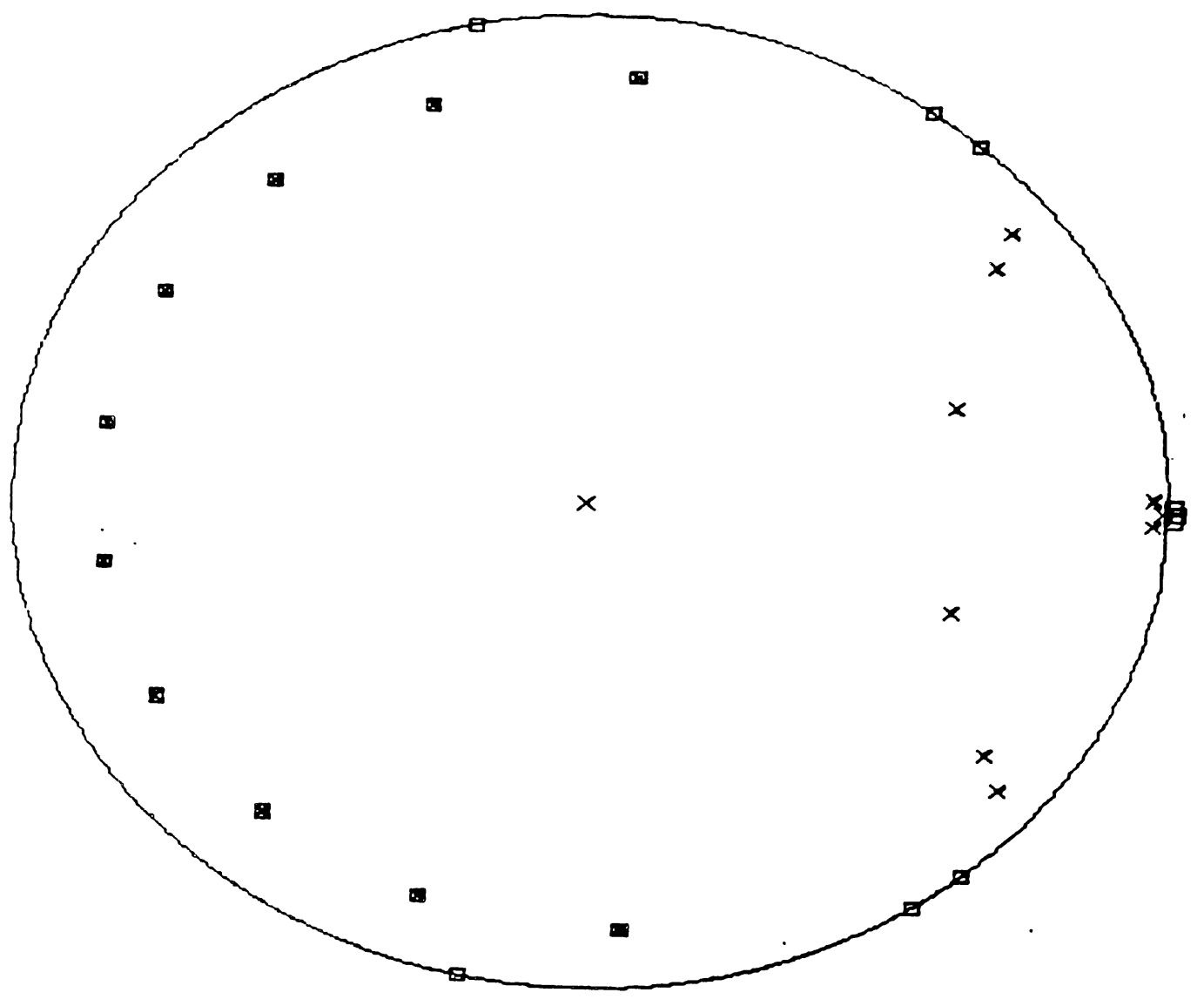

Fig. 6 Pole-Zero Distribution With Pole and Zero Overspecification

$(M=20, L=20)$ 


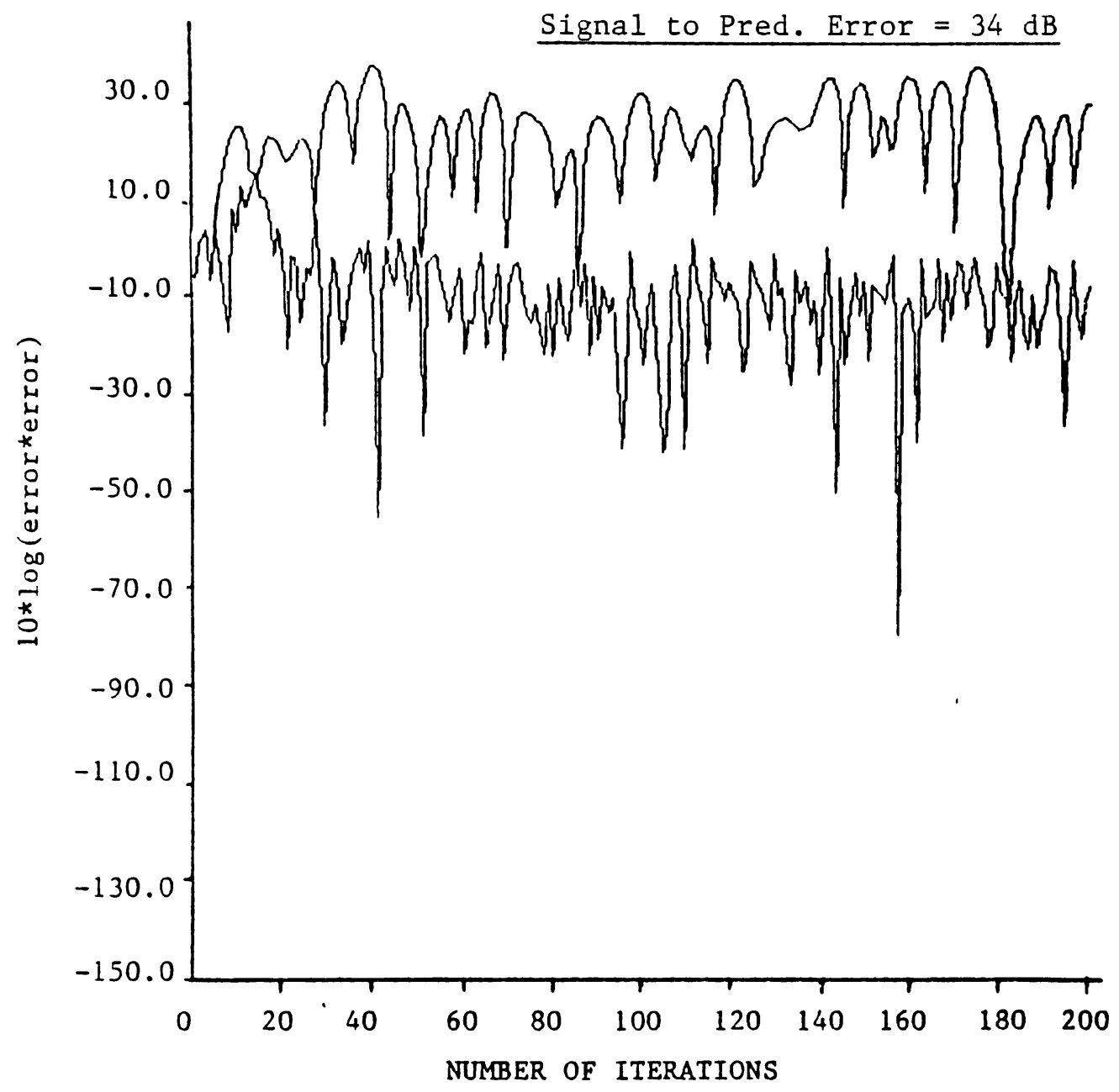

Fig. 7 Prediction Error in the Presence of Noise (rms value 0.1 )

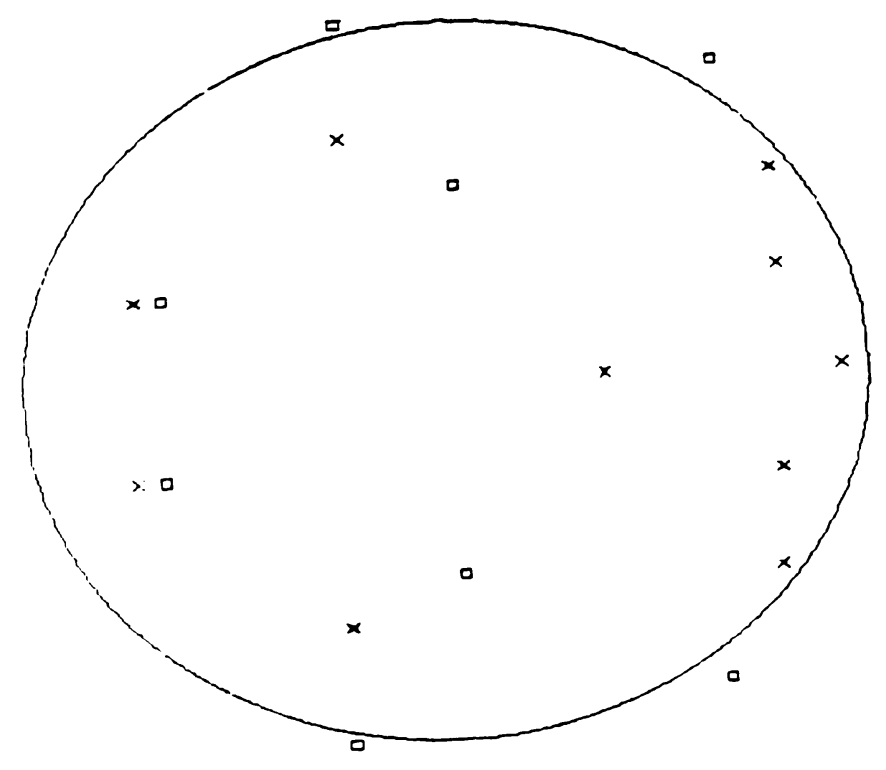

Fig. 8 Pole-Zero Distribution with Noise 


\section{Appendix}

In this appendix the projection update is derived directly from matrix methods. The projection obtained by augmenting the column space $w$ to $Z$ is,

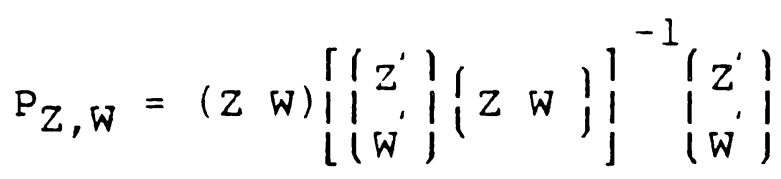

We must find an inverse such that,

$\left\{\begin{array}{ll}z z^{\prime} & z^{\prime} w \\ w^{\prime} z & w^{\prime} w\end{array}\right\}\left[\begin{array}{ll}A_{1} & A_{2} \\ B_{1} & B_{2}\end{array}\right\}=I$

Or,

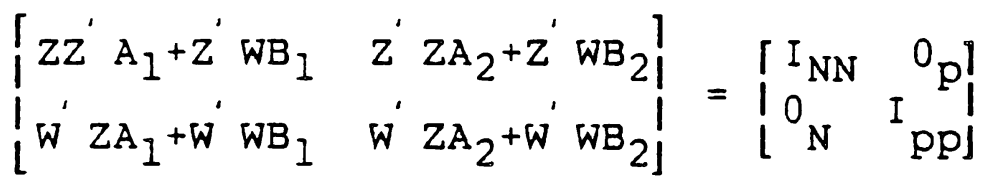

This leads to four equations:

$\mathrm{Z}^{\prime} \mathrm{ZA}_{1}+\mathrm{Z}^{\prime} \mathrm{WB}_{1}=\mathrm{I}_{\mathrm{NN}}$

$\mathrm{w}^{\prime} \mathrm{ZA_{1 }}+\mathrm{w}^{\prime} \mathrm{WB}_{1}=\mathrm{o}_{\mathrm{p}}$

$\mathrm{z}^{\prime} \mathrm{ZA_{2 }}+\mathrm{z}^{\prime} \mathrm{WB}_{2}=\mathrm{O}_{\mathrm{N}}$

$\mathrm{W}^{\prime} \mathrm{ZA}_{2}+\mathrm{W}^{\prime} \mathrm{WB}_{2}=\mathrm{I}_{\mathrm{pp}}$

From (3),

$A_{2}=-\left(z^{\prime} z\right)^{-1} z^{\prime} \mathrm{wB}_{2}$

Substituting into (4) and rearranging,

$B_{2}=\left[w^{\prime} w-w^{\prime} Z\left(z^{\prime} z\right)^{-1} z^{\prime} w\right]^{-1}$

Hence, 
$A_{2}=-\left(z^{\prime} z\right)^{-1} z^{\prime} w\left[w w^{\prime}-w^{\prime} z\left(z^{\prime} z\right)^{-1} z^{\prime} w\right]^{-1}$

Similarly we obtain,

$B_{1}=\left[w^{\prime} w-w^{\prime} z\left(z^{\prime} z\right)^{-1} z^{\prime} w\right]^{-1}\left(w^{\prime} z\right)\left(z^{\prime} z\right)^{-1}$

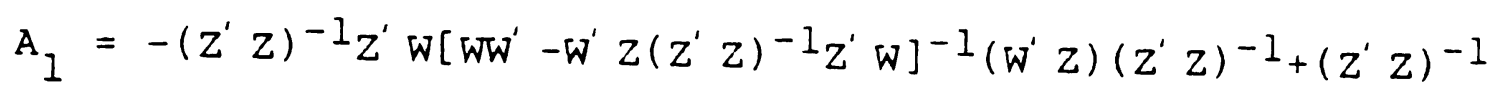

Substituting the inverse into the projection update and after some algebra we obtain,

$\mathrm{P}_{Z, W}=\mathrm{P}_{Z}+\mathrm{P}_{Z^{\prime}}^{\mid} \mathrm{W}\left(\mathrm{W}^{\prime} \mathrm{P}_{Z^{\prime}}^{\mid}\right)^{-1} \mathrm{~W}^{\prime} \mathrm{P}_{Z} \mid$

Note that,

$P_{Z}^{l}=I-P_{Z}$

The above relationships could have been derived using the generalized inverses. It is interesting to note that if we define,

$$
\mathrm{V}=\mathrm{P}_{\mathrm{Z}}^{\mathrm{W}}
$$

Then the projection update (5) can be written,

$P_{Z, W}=P_{Z}+P_{V}$

In fact an alternative derivation of (5) is suggested by (7). The vector $V$ represents that part of $W$ that cannot be estimated by the space spanned by the columns of $Z$. It is orthogonal to the sub space $z$. Hence the projection operator that includes the subspace $Z$ and $W$ can be expressed as a direct sum of the projection operator of the subspace $Z\left(\mathrm{P}_{Z}\right.$ ) and the projection operator $P_{V}$ of the subspace spanned by $V$ which is orthogonal to $Z$. Thus,

$$
P_{Z, W}=P_{Z}+P_{V}
$$




\section{REFERENCES}

[1] D.D. Falconer and L. Ljung, "Application of Fast Kalman Estimation to Adaptive Equalization," IEEE Transactions on Communications, vol. COM-26, pp. pp. 1439-1446., october 1978 .

[2] John M. Cioffi and Thomas Kailath, "Fast, RecursiveLeast-Squares Transversal Filters for Adaptive Filtering," IEEE Transactions on Acoustics, Speech, and Signal Processing, vol. ASSP-32, no. 2, pp.pp.

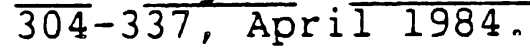

[3] L. Ljung, M. Morf, and D. D. Falconer, "Fast Calculation of Gain Matrices for Recursive Estimation Schemes," Int. Journal of Control, vol. 27, no. I, pp. pp. 1- $\overline{19}, 1 \overline{978 . ~}$

[4] Sasan Ardalan and S. T. Alexander, "Rapid Echo Cancellation Using Fast Kalman Pole-Zero Modeling," IEEE Transactions on Communications, Submitted.

[5] Sasan Ardalan, "Pole/Zero Echo Path Modeling and Recursive Least Squares Echo Cancellation," IEEE Workshop On Digital Signal Processing, October $19 \overline{84}$.

[6] Sasan Ardalan, "Pole/Zero Fast Kalman Echo Cancellation and Application to Actual Measured Echo," International Conference on Communications, ICC-85, June 1985 .

[7] Daniel T. L. Lee, Martin Morf, and Benjamin Friedlander, "Recursive Least Squares Ladder Estimation Algorithms," IEEE Transactions on Acoustics,

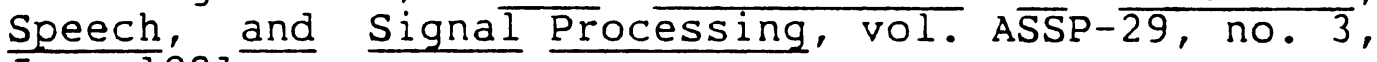
June 1981 .

[8] Boaz Porat, Benjamin Friedlander, and Martin Morf, "Square Root Covariance Ladder Algorithms," IEEE Transactions on Automatic Control, vol. AC-27, no. 4, pp. pp. 813-825, August 1982 . 